\title{
tic\&société
}

Vol. 12, $\mathrm{N}^{\circ} 1$ | 1er semestre 2018

Communs numériques et communs de la connaissance

\section{Conditions d'émergence et enjeux des communs scientifiques à partir d'une expérimentation lyonnaise}

Claire BROSSAUD

\section{CpenEdition}

Journals

\section{Édition électronique}

URL : http://journals.openedition.org/ticetsociete/2435

DOI : 10.4000/ticetsociete.2435

Éditeur

Association ARTIC

Édition imprimée

Pagination : 201-228

Référence électronique

Claire BROSSAUD, «Conditions d'émergence et enjeux des communs scientifiques à partir d'une expérimentation lyonnaise », tic\&société [En ligne], Vol. 12, № 1 | 1er semestre 2018, mis en ligne le 31 mai 2018, consulté le 10 octobre 2020. URL : http://journals.openedition.org/ticetsociete/2435 ; DOI : https://doi.org/10.4000/ticetsociete.2435 
tic\&société - 12(1), 2018

\section{Conditions d'émergence et enjeux des communs scientifiques à partir d'une expérimentation lyonnaise}

\section{Claire BROSSAUD}

Claire Brossaud est chercheure en sciences sociales associée au laboratoire EVS-LAURE (UMR 5600) de l'école d'architecture de Lyon. Elle s'intéresse aux communs de la connaissance depuis une dizaine d'années au sein de l'association VECAM et aux communs scientifiques par l'intermédiaire de l'association lyonnaise Coexiscience, qu'elle a cofondée récemment. claire.brossaud@lyon.archi.fr

Avec le concours d'Alain Mille, Professeur émérite en intelligence artificielle, LIRIS (UMR CNRS 5205), Université Lyon 


\title{
Conditions d'émergence et enjeux des communs scientifiques à partir d'une expérimentation lyonnaise
}

Résumé : L'article se propose de mettre en lumière les filiations intellectuelles et les enjeux des communs scientifiques, caractérisés ici par des activités visant à gérer collectivement et à faire perdurer des ressources informationnelles issues de la recherche. Pour ce faire, il s'appuie sur une expérimentation orientée vers la création d'un "tiers espace » de recherche et d'innovation dans l'agglomération lyonnaise. II montre d'abord comment la critique sociale fondatrice des communs s'est emparée de la science à travers le phénomène des enclosures. Puis, il insiste sur le fait que les communs scientifiques ne se limitent pas à l'approche numérique de l'open science et touchent également aux sciences participatives et citoyennes. L'article identifie enfin trois conditions de leurs possibilités: épistémologique, économique et politique. Au fil de l'analyse, les communs scientifiques apparaissent moins comme des "biens" à protéger ex nihilo que comme un processus culturel, historique et cognitif.

Mots-clés : communs scientifiques, histoire, enjeux, processus, pratiques de recherche.

\begin{abstract}
The article highlights the intellectual filiations and the issues surrounding scientific commons, characterized by activities of collectively managing and sustaining information resources resulting from the research practices. It is based on an experiment that aimed to build a shared hybrid space of research and innovation in the Lyon metropolitan area. First, it shows how the initial social criticism of the scientific commons followed the phenomenon of enclosures. Then, it stresses that they aren't limited exclusively to digital open science but are also derived from participatory and citizen sciences. Finally, the article identifies three conditions of possibility: epistemological, economic and political. Upon analysis, scientific commons
\end{abstract}


Conditions d'émergence et enjeux des communs scientifiques

à partir d'une expérimentation lyonnaise

appear less as "goods" to be protected ex nihilo than as a cultural, historical, and cognitive process.

Keywords: scientific commons, history, issues, process, research practices.

Resumen: El objetivo de este artículo es de resaltar las filiaciones intelectuales y los retos de los comunes científicos, que se caracterizan por ser actividades que persiguen la gestión de manera colectiva y por hacer perdurar los recursos de información provenientes de la comunidad de investigación. Para ello, este artículo se basa en un experimento que pretende construir un "tercer espacio " de investigación y de innovación en el área metropolitana de Lyon. En primer lugar, muestra como la crítica social de los comunes científicos se apoderó de la ciencia a través del fenómeno de los "vallamientos". Posteriormente se insiste en que los bienes comunes científicos no se limitan en un planteamiento numérico de la ciencia abierta ("Open science") sino que, al contrario, abordan también las ciencias participativas y ciudadanas. Finalmente, se identifican tres condiciones de sus posibilidades: epistemológica, económica y política. Del análisis se deprende que los comunes científicos aparecen menos como «bienes» a proteger ex nihilo que como un proceso cultural, histórico y cognitivo.

Palabras clave: communes cientificos, historia, retos, proceso, prácticas de investigación. 


\section{Introduction}

Depuis quelques années, la science représente, avec le numérique, un exemple emblématique et une opportunité de repenser la place de la production, du partage et de la diffusion de connaissances dans nos sociétés. Les communs de la connaissance scientifique ont été assez peu étudiés par les chercheurs eux-mêmes, si ce n'est précisément à travers le filtre du numérique, par l'open science (Hess et Ostrom, 2006). Jusqu'alors, ce sont surtout des approches économiques et juridiques centrées autour de la "ressource " qui ont dominé ce champ. Dans ce contexte, les codes génétiques (CookDeegan et Dedeurwaerdere, 2006), les données numériques, les savoirs traditionnels, etc., ont souvent été assimilés à des "biens" dotés de caractéristiques particulières. Cette approche, très largement inspirée des travaux d'Elinor Ostrom (1990), a donné lieu à toute une série de réflexions et d'actions visant à limiter le contrôle et l'appropriation privative des connaissances grâce à différents faisceaux de droits garantissant une gouvernance partagée de la ressource.

Aujourd'hui, on remarque que le « bien » n'est plus l'élément central du raisonnement sur les communs. Les sciences sociales ont en effet déplacé la focale vers les dynamiques sociales et l' " agir politique " dont le mouvement des communs est porteur (Bollier, 2014 ; Dardot et Laval, 2014 ; Nicolas-Le Strat, 2014). Ces approches ont montré notamment que l'engagement en faveur des communs s'appuie souvent sur des processus auto-organisés qui se construisent en situation et placent l'expérimentation pratique - plus que son résultat ou son issue - au centre. Ainsi, les formes d'organisation et de régulation des communs reposent davantage sur des actions instituantes et non plus seulement sur des catégories préétablies comme celles du droit.

L'objectif de cet article est de présenter les cadres conceptuels qui ont présidé à la mise en œuvre d'une expérimentation actuellement en cours dans l'agglomération lyonnaise. En 2016, deux chercheurs impliqués dans le mouvement des communs, rejoints en quelques mois par des 
Conditions d'émergence et enjeux des communs scientifiques

à partir d'une expérimentation lyonnaise

collègues, des artistes, des makers et des entrepreneurs, se sont lancés dans la création d'un "tiers espace scientifique » baptisé provisoirement Coexiscience. Leur volonté était de traduire en acte la notion de communs de la connaissance scientifique et d'interpeller concrètement la manière dont les pratiques des communs transforment les cadres socioéconomiques, les modèles cognitifs et les outils de production de la recherche ${ }^{1}$. Partant de cette initiative, nous déroulerons ici une réflexion générale sur les différentes orientations et incarnations des «communs scientifiques». Nous mentionnerons d'abord comment ce projet renvoie à une nécessité de décloisonner la recherche, après avoir identifié des formes d'enclosures spécifiques au champ scientifique. Nous expliquerons ensuite comment les «sciences en communs ", poussées par des mouvements issus des sciences citoyennes et de l'open science, sont aujourd'hui possibles parce que l'accès aux ressources informationnelles devient de plus en plus massif, contributif et ouvert. Cette caractéristique ouvre des perspectives d'appropriation de plus en plus importantes de la connaissance scientifique. Enfin, au-delà de cette ouverture, nous envisagerons d'autres conditions à cette appropriation : épistémologique, économique et politique.

\footnotetext{
${ }^{1}$ Coexiscience est une association ouverte à toute discipline et à tout public visant une bonne articulation entre recherche scientifique et innovation sociale et environnementale. Elle évolue vers un espace hybride de type "tiers-lieu» (incubation ; formation, recherche, conseil ; médiations, art-science-numérique), dont l'activité économique sera prise en charge par une coopérative. L'association soutient et fait émerger des projets transformateurs de la société de la connaissance ainsi que ceux qui les portent à l'échelle de la métropole de Lyon et de la région Auvergne-Rhône-Alpes: Skoli (représentation graphique et visualisation de la donnée), Géosapiens (musée itinérant sur l'anthropocène), NatBraille (logiciel libre de transcription et détranscription du braille), Demostalie (ateliers de médiation artscience en physicochimie et mathématique), etc. Coexiscience rassemble actuellement quinze membres au sein de son conseil collégial, six membres actifs et plus d'une centaine de soutiens sur une liste de diffusion.
} 


\section{La science marquée par des formes d'enclosures}

Les enclosures sont une idée fondatrice des communs, qui permettent de comprendre comment différents champs scientifiques comme les mathématiques, le vivant ou l'information, qui étaient autrefois exempts de toute forme d'appropriation, ont fait progressivement l'objet de fermetures juridiques artificielles (Hess et Ostrom, 2003). À l'origine, cette idée rend compte de la privatisation accrue des terres communales en Europe au $\mathrm{XV}^{\mathrm{e}}$ et $\mathrm{XVI}^{\mathrm{e}}$ siècles, notamment en Angleterre. Ces privatisations transforment un système de coopération et de gestion communautaire par un régime de propriété privée, marquant également la fin d'un droit d'usage et la possibilité de tirer bénéfice des sols pour les agriculteurs. En référence à cette période, James Boyle a comparé l'extension continue des droits de propriété intellectuelle sur la connaissance à un "second mouvement des enclosures " (Boyle, 2003). Depuis une dizaine d'années maintenant, les communs de la connaissance scientifique sont fondés sur une critique des droits de propriété intellectuelle tels le brevet ou le copyright. Le symbole de cette dénonciation vise l'exploitation abusive des semences ou des plantes qui ont été préalablement brevetées par des groupes industriels. Comme chacun sait en effet, il est désormais possible d'avoir des droits exclusifs sur les variétés de plantes présentant certaines caractéristiques afin de pouvoir commercialiser ensuite des produits dérivés. Ce phénomène d'appropriation du vivant par la propriété intellectuelle entraîne des conséquences dramatiques pour la préservation des savoirs traditionnels, des économies locales et de la biodiversité. Les agriculteurs qui utilisent par exemple des semences de soja ou de maïs produites par des géants de l'industrie agroalimentaires sont ainsi soumis à des conditions d'après-vente qui leur interdisent de replanter leurs propres graines et les forcent à acheter chaque année les semences brevetées ${ }^{2}$.

\footnotetext{
${ }^{2}$ Voir, à ce sujet, le film de Carole Poliquin, Le bien commun, l'assaut final (2003).
} 
Conditions d'émergence et enjeux des communs scientifiques

à partir d'une expérimentation lyonnaise

\subsection{Les caractéristiques des enclosures sur les communs de la connaissance}

Le Crosnier (2015) distingue trois formes d'enclosures spécifiques aux communs de la connaissance. Tout d'abord, celles qui pèsent sur les ressources communes elles-mêmes il place dans cette catégorie les possibilités de fraude et d'autres pollutions (les anti-commons) qui érodent la confiance collective dans la recherche. En France, les zones à régime restrictif (ZRR) visent à protéger réglementairement des installations publiques, du matériel, des recherches ou des fabrications afin de limiter précisément le pillage ou des exploitations malveillantes. Ensuite, il y a les enclosures qui menacent les communautés qui ont construit et qui maintiennent des ressources en organisant leurs usages partagés - tel est le cas d'une communauté qui détient un savoir traditionnel et qu'on empêche de l'utiliser en raison d'un brevet. Enfin, il y a les enclosures qui menacent l'activité même de mise en commun - limiter le partage du code et des algorithmes par les développeurs pour la mise en œuvre de logiciels.

Ce même auteur explique par ailleurs que l'origine de ces enclosures relève de trois catégories distinctes : les restrictions légales, les interventions technologiques et les décisions économiques. La première catégorie abonde largement la littérature sur les communs de la connaissance scientifique et détermine le cadre réglementaire de l'exploitation de la ressource. La seconde catégorie est une forme de fermeture intégrée à la ressource elle-même. Elle affecte beaucoup la recherche sur le vivant avec les OGM (organismes génétiquement modifiés) ou les GURTs (Genetic Use Restriction Technologies) qui, par exemple, bloquent certains traits dans les plantes et notamment leur capacité de reproduction. Des modèles d'affaires peuvent également amener à limiter l'accès à la ressource. L'agriculture industrielle, notamment, est tentée de consolider sa position sur un marché en produisant des fruits et des légumes calibrés, qui exigent eux-mêmes des semences certifiées, qui, à leur tour, nécessitent l'achat de produits chimiques spécifiques (Le Crosnier, 2015).

Ce dernier processus invite à considérer plus avant une 
autre critique des communs de la connaissance scientifique à l'adresse d'une économie dominée par le capitalisme informationnel (Castells, 1998) ou cognitif (Moulier-Boutang, 2007). Cette notion souligne que nous sommes entrés, depuis quelques décennies, dans une troisième phase du capitalisme où l'accumulation économique est centrée sur les actifs immatériels. L'objectif du capitalisme cognitif serait de capter les externalités positives telles que l'open innovation ou l'intelligence collective, principalement dans les secteurs numériques et scientifiques. La pharmacie, l'agroalimentaire et l'économie collaborative sont particulièrement concernés. Ici, la principale source de valeur marchande est le travail immatériel, et non la main-d'œuvre et les machines comme au $\mathrm{XIX}^{\mathrm{e}}$ siècle. Pour certains auteurs, les dérives qui en découlent conduisent à l'émergence du digital labor et d'une nouvelle classe de travailleurs pauvres (Cardon et Casilli, 2015) consistant à produire une valeur d'usage d'un service (la mise à disposition d'une voiture et d'un chauffeur via une plateforme collaborative par exemple) ou d'un bien sans pouvoir réellement agir sur sa valeur d'échange (seuls les propriétaires de ces plateformes d'intermédiation profitent directement des avantages financiers liés à cette mise à disposition). Le capitalisme cognitif est ainsi associé à un accaparement des communs de l'information et de la connaissance.

\subsection{Les effets de ces enclosures sur les conditions de production et de diffusion de la recherche}

Sachant que la recherche, en tant que secteur d'activité socioéconomique, représente le vecteur opérationnel de la science, il importe désormais de resituer les effets de ces enclosures sur ses conditions de production et de diffusion. Quatre impacts affectent aujourd'hui les politiques nationales et internationales de recherche et d'innovation. Le premier d'entre eux concerne l'application des titres de propriété intellectuelle, comme le brevet, dont les demandes augmentent de manière exponentielle depuis 20 ans (Bernard, 2015). En 2016, 3,1 millions de demandes de brevets ont été déposées dans le monde, soit $8,3 \%$ de plus qu'en 2015 et 240600 demandes supplémentaires (dont 236600 par la Chine). Cela représente 
Conditions d'émergence et enjeux des communs scientifiques

à partir d'une expérimentation lyonnaise

près de six demandes de brevets par minute ${ }^{3}$. La création des centres de transfert de technologies dans la plupart des métropoles occidentales depuis vingt ans, permettant de faire passer des résultats de la recherche publique vers le marché sous la forme de droit de propriété intellectuelle et d'actifs financiers, a accéléré ce processus (en France, 1184 brevets ont été produits par les SATT [Société d'accélération et de transfert de technologie] depuis cinq ans ${ }^{4}$ ).

Le second impact est aussi connu que le premier. II est lié à l'industrie de l'édition scientifique, dont le modèle d'affaires, qui prévaut depuis le début du $\mathrm{XX}^{\mathrm{e}}$ siècle, est basé sur des pratiques commerciales entre les bibliothèques et les éditeurs pour la vente et l'achat de contenu. Celui-ci fait porter simultanément ses coûts d'abonnement aux revues sur des centres de documentation universitaires publics et ses coûts de production, sur les chercheurs financés également par des fonds publics. Parallèlement, on assiste à une concentration et à une marchandisation de plus en plus forte du secteur. II ne resterait aujourd'hui que cinq à six majors très lucratives et un nombre très important de revues indépendantes qui peinent à trouver leur autonomie financière. Afin de protester contre les prix de souscription élevés des éditeurs traditionnels, des plateformes numériques de libre accès à l'information scientifique n'ont cessé de se développer depuis une quinzaine d'années.

Le troisième impact des enclosures précitées porte sur le financement de la recherche. Depuis plus d'une vingtaine d'années, sur le plan national comme sur le plan international, les opérateurs publics ont donné une place centrale aux modes de financement de la recherche par projet, jusqu'alors marginaux dans de nombreuses disciplines. Ce processus a eu d'autres conséquences que celles pour lesquelles il avait été inventé, à savoir l'optimisation des investissements : accentuation des partenariats public/privé, soumission des projets de recherche à des conditions de résultats économiques

\footnotetext{
${ }^{3}$ Planétoscope (s. d.). Nombre de demandes de brevets délivrés par l'OMPI. Repéré https://www.planetoscope.com/Etonnant/353-nombre-de-demandes-de-brevetsdelivres-par-l-ompi.html. Voir aussi les statistiques de l'Organisation mondiale de la propriété intellectuelle (OMPI) : http://www.wipo.int/ipstats/fr/.

${ }^{4}$ Ces données (2016) sont tirées des Sociétés d'accélération du transfert de technologies (http://www.satt.fr/).
} 
à court terme, perte de repère d'une partie de la profession qui ne maîtrise plus les finalités ultimes de leur métier, augmentation substantielle des emplois temporaires et précaires, baisse d'autonomie des acteurs scientifiques et de la puissance publique et captation de la production du savoir et de sa valeur d'usage à des fins mercantiles. Fers de lance de l'open innovation, les fab labs et les living labs sont désormais intégrés aux établissements d'enseignement supérieur dans la perspective de promouvoir cet objectif.

Le quatrième impact a trait aux formes de régulation et de légitimation professionnelle des chercheurs. De plus en plus de normes, de classifications, de critères de validation ou d'évaluation de la production scientifique sont fixés par des communautés de chercheurs (ou de pairs) qui officient dans des instances nationales ou internationales: comité d'experts posant les limites de la science, comité scientifique définissant les programmes de recherche, comité de rédaction précisant des règles de publications... Cette validation varie d'une communauté de recherche à l'autre et se justifie par le fait qu'une connaissance peut être considérée comme "scientifique » en fonction d'une épistémologie propre à chaque discipline. Au sein de ces spécialités, la notion d'excellence est fondée sur des indicateurs favorisant la mesure de l'activité de production et de dissémination de la connaissance afin d'évaluer la réputation d'une école, par exemple. En guise d'illustration, on retiendra la forte pression à la publication (" publish or perish ») sur certains titres de revue à fort impact. Pour ce faire, des indicateurs bibliométriques faisant mention de citations de ces titres dans les articles sont utilisés. On constate aussi une intériorisation de contraintes de publication, telle une prédilection pour la langue anglaise ou pour des « design hypothético-déductifs assortis d'une méthode quantitative " (Lebouc et Chartier, 2015, parag. 51). De ces différentes conventions, il résulte un régime de "coopétition " larvé entre collègues, en même temps qu'un réel mal-être de la profession. II en découle également une défiance de plus en plus grande de certains publics vis-à-vis du monde de la recherche, celui-ci étant accusé tantôt d'élitisme tantôt de corporatisme, ou bien de ne plus servir l'intérêt général.

D'aucuns l'auront deviné à l'issue de cette première partie de la démonstration, le projet Coexiscience se veut être une 
Conditions d'émergence et enjeux des communs scientifiques

à partir d'une expérimentation lyonnaise

alternative aux logiques néolibérales d'un côté, suspectées de s'approprier des pans entiers de l'activité de recherche et de rendre ainsi des ressources cognitives inaccessibles à tous, et une prise de distance avec les cadres modernistes organisés et institués de la recherche actuelle de l'autre. Si la première partie de notre exposé visait à déconstruire ces cadres de références, la seconde a pour ambition de présenter des formes alternatives de production et de diffusion de la connaissance propices au développement de communs scientifiques.

\section{Des formes de production et de diffusion des sciences et des techniques de plus en plus ouvertes et contributives}

Alors que les institutions se ferment et se bureaucratisent, la société, de son côté, se montre particulièrement intéressée par la recherche et l'éducation aux sciences. Les indices de cet intérêt sont nombreux : l'étonnant succès des MOOCs (Massive Open Online Courses), avec des millions d'inscrits dans le monde, démontre une appétence pour un accès à des savoirs ouverts et interactifs ; les makerspace citoyens, voire certaines friches artistiques, se développent peu à peu selon des modalités de production scientifiques et techniques " artisanales »; le succès et la confiance dans les pages scientifiques de Wikipédia s'affirment un peu plus chaque jour ; de nombreux blogues et de nombreuses plateformes de collectes de données fleurissent; les associations d'ouverture de la science se multiplient; le succès des magazines de vulgarisation scientifique et de la fête de la science est attesté...

La pénétration de la recherche par la société civile n'est pas nouvelle et puise dans l'histoire des sciences elles-mêmes. Deux filiations semblent dessiner aujourd'hui les contours des communs scientifiques: les sciences citoyennes et l'open science. Ces héritages indiquent chacun à leur manière que, parallèlement aux renforcements des enclosures précédemment évoquées, l'on assiste à l'émergence de formes de production et de diffusion des sciences et des techniques de plus en plus ouvertes et contributives. 


\subsection{Les " sciences en communs " au carrefour des sciences citoyennes et de l'open science}

Depuis une centaine d'années environ, les sciences citoyennes mettent en doute le positivisme, le rationalisme et l'obscurantisme de la science. Trois voies se sont dégagées afin d'enrayer ces évolutions. La première promeut un accès éthique à la connaissance scientifique. Pour ce courant, il importe que des considérations sociales et environnementales imprègnent les choix scientifiques et techniques, et ce, afin de faire face aux dérives d'une technoscience qui, au moyen d'une humanité augmentée par exemple, serait capable de gérer les défis du monde actuel, qu'ils soient écologiques, économiques, sociaux, etc. (Feenberg, 2010 ; Testart, 2015). Pour ce faire, le débat démocratique est préconisé dans les institutions de recherche, et ce, à différentes échelles de décision, afin d'élaborer ensemble des normes sociotechniques. Les partisans d'une démocratie scientifique et technique affirment, par exemple, qu'il convient d'inventer de nouvelles procédures " dialogiques », telles des conférences ou des conventions de citoyens, lorsque des controverses adviennent sur des sujets sensibles comme les déchets nucléaires, les organismes génétiquement modifiés, l'amiante, etc. (Callon, Lascoumes et Barthe, 2001.) Ils défendent également une exigence de responsabilité individuelle et collective vis-à-vis de la science (Pellé et Reber, 2016) que l'on retrouve notamment dans des actions visant à protéger les lanceurs d'alerte. On peut aussi rattacher à cette exigence éthique le mouvement des slow sciences, "pour qui l'idée n'est pas de faire du ralentissement une nouvelle norme qui remplacerait celle de l'accélération mais plutôt de respecter une forme de chrono-diversité inhérente à l'élaboration des savoirs et où la lenteur aurait toute sa place » (Coutellec, 2012, p. 3).

Au sein des sciences dites " citoyennes ", le second courant est incarné par les sciences participatives. II affirme que la recherche doit être coproduite par la société à l'aide de différentes démarches intégratives. La recherche-action, la recherche partenariale, la recherche-intervention impliquent généralement l'usager dans les protocoles de recueil de données et, à de plus rares exceptions, dans la définition des problématiques et les analyses (Storup, Millot et Neubauer, 2013). Ces démarches proposent beaucoup de méthodes et 
Conditions d'émergence et enjeux des communs scientifiques

à partir d'une expérimentation lyonnaise

d'outils empiriques issus de différentes disciplines où chercheurs et acteurs sont simultanément impliqués dans l'expérience scientifique. Les approches pragmatiques anglosaxonnes en sciences humaines et sociales incarnent cette tendance : l'École de Chicago en sociologie, les cultural studies en anthropologie, etc. Une partie de l'agronomie, les sciences pour le développement et les sciences de l'environnement ont également une longue tradition de recherche participative. Ces " écoles " ne revendiquent pas une neutralité axiologique pour affirmer l'objectivité de la science, mais visent au contraire un partage des savoirs et des pouvoirs au moyen d'une autre épistémologie (Coutellec, 2015). Elles soutiennent également une production de la recherche souvent centrée sur une dynamique collective et sur la pluralité scientifique.

Enfin, le courant de la médiation scientifique s'est imposé ces dernières années pour réduire les distances entre les chercheurs et leurs publics. Jusque dans les années 1970, on parlait de "popularisation » ou de "vulgarisation » des sciences selon un format de transmission des connaissances descendant du chercheur vers l'usager. Avec la médiation, le public n'est pas seulement informé en mode "push », il est un partenaire de la relation touchant à la communication scientifique, voire une partie prenante du dialogue sciencesociété. Beaucoup de programmes et d'institutions ont été créés depuis environ 20 ans afin de favoriser ce dialogue : les boutiques de sciences en Europe, les CCSTI (Centre de culture scientifique et technique) en France, etc.

Le second héritage des communs scientifiques puise ses sources dans les communs numériques. Ce que nous appelons l'open science se limite ici aux liens de causalité que la recherche entretient avec la sphère digitale et renvoie donc à une acception assez récente ${ }^{5}$. "Bien que le terme de "sciences ouvertes" soit parfois critiqué et qu'il désigne des pratiques disparates, il permet néanmoins de cerner un certain désir de renouvellement des pratiques scientifiques qui s'inscrivent dans la filiation des communs numériques »(Couture, 2014, s. p.).

\footnotetext{
${ }^{5}$ De nombreux auteurs font remonter la science ouverte après 1945, notamment aux travaux de R.K. Merton, qui promouvait de nouvelles normes basées sur l'interdisciplinarité, l'abandon de la propriété intellectuelle au bénéfice de la coopération scientifique et les débuts de la documentation (cf. Chesbrough, 2015).
} 
Ce courant met l'accent sur les restrictions portées à la libre circulation des informations scientifiques, comme en témoignent les mouvements pour l'accès ouvert aux publications et aux données issues de la recherche (Open Archive Initiative [OAI] à l'échelle internationale, Science commons, Open Knowledge Foundation Network [OKFN] aux États-Unis, association Science et biens communs au Québec, Savoirs communs et VECAM en France, mais aussi toutes les licences rendant possible cet accès, comme les licences Creative Commons, la Free Documentation License (FDL), l'Open Content Principles, I'Open Publication Licenses et la Public Library of Science Open Access License). Pour Delfanty (2013), les sciences ouvertes concernent l'accès ouvert aux publications scientifiques et aux données, les nouvelles formes de production scientifique (blogues, wiki, vidéo, MOOCs, etc.), la participation citoyenne à la production scientifique, notamment à la collecte et à l'analyse des données ${ }^{6}$, et, enfin, les altmetrics ${ }^{7}$ permettant d'obtenir des mesures de l'impact de la recherche à l'extérieur du milieu académique indépendamment des traditionnelles citations d'auteurs ou d'articles.

\subsection{L'utilisateur accède au statut d'agent producteur, transformateur d'informations scientifiques}

Plus encore qu'avec les sciences participatives, les sciences ouvertes ont permis de faire potentiellement passer l'usager de la recherche au statut d'acteur de la recherche, en lui fournissant l'occasion de participer à sa production, par l'intermédiaire de l'open data, et à sa dissémination, grâce au libre accès. Cela a été rendu possible parce que l'informatique permet désormais d'utiliser, de réutiliser et redistribuer l'information scientifique sans restriction juridique, technologique ou sociale. La généralisation de l'accès à Internet et le développement du web ont en effet modifié

${ }^{6}$ De nombreuses expériences indépendantes d'initiatives publiques existent aujourd'hui afin de capter des données - essentiellement dans le domaine de l'environnement - qui traduisent de nouvelles formes de coopération entre chercheurs et citoyens/habitants. C'est le cas, par exemple, du très célèbre site web Tela Botanica, qui rassemble scientifiques et amateurs autour de la collecte de données en botanique.

${ }^{7}$ Voir le manifeste altmetrics (http://altmetrics.org/manifesto/). 
Conditions d'émergence et enjeux des communs scientifiques

à partir d'une expérimentation lyonnaise

considérablement la relation de tout un chacun à la connaissance en passant de l'idée d'utilisation d'outils informatiques pour achever telle ou telle tâche à l'idée de réaliser ses activités au sein d'un environnement numérique. Le web a d'abord été documentaire avant de se transformer en web des données, ce qu'il est massivement aujourd'hui. Dans ce contexte, l'utilisateur devient un agent humain au même titre qu'il existe toute une panoplie d'agents artificiels, intégrés dans l'environnement numérique et agissant sur les flux de données pour automatiser des tâches en relation avec son activité, mais aussi pour exécuter des algorithmes destinés à réaliser des objectifs de fournisseurs de services. D'ailleurs, le rôle d'agentutilisateur lui échappe très largement au profit de ces mêmes fournisseurs de service qui, de plus en plus, éditorialisent des données résultant du traitement des interactions entre pairs (Mille et Magué, 2012).

Vue sous cet angle, l'idée qu'il suffit d'ouvrir des données scientifiques sur des terminaux de plus en plus attractifs pour qu'elles puissent être collectées et réutilisées par d'autres, comme le sont déjà les open data centers dans le secteur du numérique, apparaît inepte à de nombreux scientifiques. Comme le précise Bruno Latour, les données sont toujours des " obtenues », c'est-à-dire qu'elles résultent déjà d'une activité réflexive, et l'utilisateur-agent, qu'il soit profane ou chercheur, ne peut-être assimilé à un simple "chasseur-cueilleur d'informations $»$, exempt de toute faculté interprétative (Fayet, 2013). Pis, l'idée que la science ne puisse vivre que de ses appareils (lieu de stockage et d'archivage de données qualitatives et quantitatives, centre de calculs et de traitement, etc.) et de ses algorithmes « rappelle une automatisation généralisée de la société dans lequel le data mining constituerait le grand récit. Or, nous savons aujourd'hui combien sont nouées formes de pouvoir et raison algorithmique » (Noyer et Carmes, $2014^{8}$ ). Le fait qu'un environnement soit ouvert et contributif peut donc conduire aussi à un nouveau positivisme et ne garantit aucunement un réel partage de la ressource informationnelle. Par ressource informationnelle, il faut entendre, ici, toutes les activités

\footnotetext{
${ }^{8}$ Citation extraite d'une version en prépublication de l'article cité en référence, actuellement non disponible en ligne (Noyer-Carmes, 2014)
} 


\section{Claire BROSSAUD}

nécessaires au traitement scientifique de l'information et non pas seulement de simples entrepôts: collecte, analyse, stockage, conservation, utilisation, valorisation, circulation, évaluation, médiation, etc. "Dans cette perspective, la conception de système de ressources informationnelles communes a pour but de permettre l'émergence et l'intelligence informationnelle » (Penaranda, Amabile et Haller, 2017, p. 6)

Les sciences ouvertes et les sciences citoyennes ont montré historiquement que le partage de l'information scientifique et technique les traverse, les premières en favorisant son libre accès sur le web, les secondes en stimulant son appropriation démocratique sous différentes formes (convention, participation, médiation). Si ces deux mouvements sont féconds pour les communs scientifiques, ils ne suffisent cependant pas à caractériser les conditions d'une gestion collective de la ressource informationnelle à l'échelle d'une communauté de pratique.

\section{De quelques conditions pour gérer en " communs » la ressource informationnelle scientifique}

Les travaux fondateurs d'Ostrom ont montré que les biens communs (ou simplement "communs") réunissaient trois éléments :

une ressource gérée hors du régime de propriété publique ou privée, une communauté, petite ou grande, localisée ou déterritorialisée, qui porte la gestion de cette ressource, l'utilise, la mobilise dans ses pratiques; une gouvernance définie par cette communauté, qui permet de protéger et le cas échéant de faire fructifier la dite [sic] ressource " (Peugeot, 2016, s. p.).

Relayée par un certain nombre d'études en gestion des systèmes d'information, en économie politique ou bien en ingénierie des connaissances, l'analyse des pratiques de coopération dans les communautés cognitives auto-organisées a déjà révélé, par ailleurs, qu'il existait différentes conditions favorisant plus ou moins l'appropriation de la ressource informationnelle. Pour ce qui concerne le projet Coexiscience, nous avons considéré que trois d'entre elles s'invitaient au 
Conditions d'émergence et enjeux des communs scientifiques

à partir d'une expérimentation lyonnaise

démarrage de l'expérimentation. La première relève d'une épistémologie des connaissances et se propose de faire du processus de fabrication de la recherche un "commun » à part entière. La seconde, plus économique, implique la mise en place de règles d'accès et d'usage de la ressource afin de favoriser son partage et de limiter son impact négatif pour la société. Enfin, la troisième est politique et a trait aux moyens organisationnels dont la communauté se dote pour gérer ensemble la ressource.

\subsection{Coconstruire le processus de fabrication de la ressource informationnelle}

Nous avons vu que les informations scientifiques, qu'elles soient données, archives, publications ou contenus pédagogiques, deviennent de plus en plus contributives et ouvertes. Avec le numérique, il est désormais possible de réexploiter des données en créant de nouvelles formes de classification (typologies, index, tableaux, etc.). En présentiel, il est également envisageable de mobiliser différentes techniques et méthodes en intelligence collective visant à solliciter l'observation, le jugement et la participation de l'usager à la recherche d'une part, par les sciences participatives, et à l'innovation d'autre part, par des outils de créativité plus ou moins connus, comme le design.

La mise en commun d'informations scientifiques permet désormais de consulter et de produire, de partager et de diffuser collectivement du savoir (Vecam, 2011), mais elle autorise aussi simultanément des représentations de situations, des itérations et des prises de décision mutualisées. Ces différents processus impliquent un niveau d'engagement et de coopération a minima dans un collectif de travail ou d'activité (De Moor, 2013). La capacité de la ressource informationnelle à perdurer n'est pas liée ici à ses propriétés intrinsèques (sa volatilité, sa cumulativité, etc.) ou à la qualité de ses supports (infrastructures de stockage, de traitement, etc.), mais au processus collaboratif dans lequel elle s'inscrit. L'expérience de l'utilisateur-acteur engagé dans une activité de recherche ou d'innovation devient alors réflexive si, tout au long de cette chaîne opératoire, ses manipulations sont connues de tous et si les mécanismes de documentarisation qui leur sont associés 
sont visibles. Par exemple, des données deviendront « corpus » parce qu'on les aura inscrites au préalable dans une réalité sociale. Une information sur Wikipédia prendra sens par rapport à une autre en lien à la finalité pédagogique d'un contenu, etc.

Ce qui intéresse ici la production d'un commun scientifique est la possibilité de décrire et de faire vivre les transformations de la ressource informationnelle: celles des collectifs qui prennent part aux expérimentations (leurs narrations, leurs débats, leurs rapports de force, etc.) ; celles des mouvements ou des trajectoires des participants et les événements qui permettent d'en témoigner; celles des différentes étapes de fabrication et de validation des savoirs à l'œuvre dans la coproduction (la définition d'une problématique, la saisie collaborative de l'information, sa vérification, les raisonnements qui étayent chaque opération de transformation et dont il importe de remonter la source, etc.). Les fondements théoriques d'une telle approche réflexive dans un environnement numérique support d'activités scientifiques sont bien établis dans la littérature, et ce, dans différentes disciplines (Brossaud et Reber, 2010 ; Noyer et Carmes, 2014). On retiendra notamment, à cet endroit, la notion de traces d'activités, que l'on retrouve aussi bien en ergonomie, en histoire ou en intelligence artificielle. Celle-ci révèle des processus d'apprentissage ou d'ingénierie des connaissances à l'œuvre dans la collaboration (Mille, 2013). De telles approches réflexives ont également été explicitées au milieu du $X X^{\mathrm{e}}$ siècle autour des démarches participatives en anthropologie (LéviStrauss), en ethnologie (Mead, Leiris), en pédagogie (Piaget), pour ne citer que ces exemples. Enfin, leurs ressorts politiques culturels sont également bien couverts par les acteurs de la société civile . $^{9}$

Toutes ces démarches marquent l'advenue de modes d'écritures visant à prendre en compte la processualité et les hétérogenèses des agencements collectifs de la recherche (Carmes et Noyey, 2014) et de l'innovation. On retrouve ici « le travail du commun " cher à Nicolas-Le Strat (2015), où se

\footnotetext{
${ }^{9}$ Cf. Ministère de la Culture et de la Communication, France (2015-2016). Sciences et techniques, une culture à partager. Culture et recherche Web, automnehiver(132).
} 
Conditions d'émergence et enjeux des communs scientifiques

à partir d'une expérimentation lyonnaise

négocient l'usage et le partage d'une ressource à travers des relations humaines, sociales ou politiques, des transactions culturelles et une coproduction de savoir-faire. L'idée d'une "science en action " n'est pas nouvelle et signifie que langages et outils, d'un côté, technique et science, d'un autre côté, sont intrinsèquement mêlés dans la production de la connaissance (Latour, 1988 ; Leroi-Gourhan, 1964). Cette posture intellectuelle met à mal notre rationalité occidentale, notamment en France, où l'espace légitime scientifique s'est construit de manière cartésienne et dialogique, grâce au prima de la raison, d'une part (notamment à partir d'une séparation radicale entre sujet et objet de la connaissance), et selon un découpage monodisciplinaire, d'autre part, dont on sait qu'il freine l'hétérodoxie des savoirs. Le dessein des communs scientifiques à cet endroit consisterait-il à réconcilier une pensée analytique - le raisonnement est dirigé consciemment, il est focalisé sur un problème ou une hypothèse impliquant ensuite une solution - et une pensée analogique ou inductive le raisonnement tire des lois générales provenant de comparaisons ou d'observations issues du réel ?

Faire de la science un objet appropriable par le plus grand nombre représente aujourd'hui un défi sociétal tant par les enjeux réflexifs et pédagogiques que par les asymétries de savoirs et de pouvoirs que cela soulève auprès d'une diversité de publics (Leclerc, 2017). L'environnement numérique, parce qu'il permet la diffusion et l'ouverture, la réutilisation et le partage de l'information, joue un rôle clé dans ce dispositif, à condition cependant qu'il soit intégré au cycle de vie de la recherche ou de l'innovation. Mais il ne saurait être le seul. Les communs scientifiques interrogent en effet en profondeur la capacité de la société à produire ses propres modèles de production et de diffusion de la connaissance.

\subsection{Favoriser l'émergence d'un « tiers espace » de production de la recherche}

Le fait que quiconque puisse potentiellement accéder au statut d'agent producteur et transformateur d'informations modifie considérablement le champ productif de la recherche et de l'innovation, comme en témoignent déjà de nombreux tiers lieux d'innovation. Les makerspace ou les fab labs citoyens, par 
exemple, sont des espaces d'expérimentation qui, souvent à partir de technologies numériques, permettent de créer et de fabriquer des objets en commun. Au-delà de la rhétorique " auto-entrepreneuriale " qu'ils véhiculent, ces espaces représentent une forme de projet politique: " lls semblent proposer une voie de réappropriation des outils et des activités de production sous une forme qui permette de surcroît de rendre actifs les utilisateurs " (Rumpala, 2014, s. p.). Les living labs improvisés ou les friches artistiques, visant à utiliser des espaces publics souvent délaissés ou promus à des projets d'infrastructures faramineux, constituent eux aussi des lieux d'innovation sociale et environnementale qui réinventent le droit à la ville (Brossaud et Fiori, 2018). Ces projets nous rappellent le caractère libertaire des sciences participatives en France, qui, contrairement au Québec notamment, se sont structurées historiquement en petites associations qui vivent souvent en marge des laboratoires publics sur des niches thématiques (Brossaud, 2013). Toutefois, l'ensemble de ces initiatives ambitionne rarement de transformer les cadres de l'économie de la connaissance que nous avons décrits dans la première partie. Cette capacité repose en effet sur la possibilité d'organiser les modalités d'une action collective permettant de faire évoluer le marché de la connaissance.

Le premier changement consisterait à privilégier la valeur d'usage de la ressource informationnelle sur sa valeur d'échange. Sans exclure des formes d'exploitation privative de la recherche, cette approche a conduit à redéfinir les cadres de propriété intellectuelle qui garantissent des droits d'accès spécifiques pour le maintien de cette ressource, tels que définis par Ostrom (Hess et Ostrom, 2006) Les licences Creative Commons et, d'une manière générale, toutes les licences contributives constituent de ce point de vue des leviers juridiques indispensables à activer afin de contrebalancer toute forme d'appropriation exclusive des résultats de recherche. Pourtant, le maintien de cette ressource, rappelle Ostrom, ne résulte pas seulement d'une organisation de la distribution des droits de propriété, mais aussi de la possibilité d'en réguler l'accès à un niveau collectif, tel un antidote à la tragédie des communs décrite par Garett Hardin.

Comment s'assurer que la production de la recherche contribue à préserver les équilibres sociaux et écologiques (le 
Conditions d'émergence et enjeux des communs scientifiques

à partir d'une expérimentation lyonnaise

vivant, la biodiversité, etc.) par d'autres formes de souveraineté que la loi ? Ostrom précise qu'il convient de définir des règles de gestion et d'exclusion de l'usage de la ressource et suggère de limiter le droit de prélèvement de la ressource pour assurer sa pérennité : nombre de vaches allant paître dans un champ par exemple, quota de pêche défini par des marins sur une zone prescrite, etc. Cependant, cette limitation reste valable pour une ressource naturelle dite "soustractive", que la consommation détruit inévitablement. La connaissance, produit intangible par excellence, est additive: elle croît en étant partagée. Sa mesure d'impact n'en est pas moins possible si l'on considère l'ensemble des outils de pilotage qui existent actuellement afin de limiter les externalités négatives de l'économie. On les trouve dans différents secteurs d'activités: indicateurs RSE (responsabilité sociale des entreprises), critères d'utilité sociale et environnementale en économie sociale et solidaire et, en germe actuellement dans le monde de la recherche, les critères $\mathrm{RRI}$ (Responsible Research Innovation) et les altmetrics précédemment évoqués. Toutefois, ces outils de mesure, appliqués ex nihilo comme de nouveaux indicateurs de performance d'une économie de la connaissance plus verte ou plus sociale, n'auront que peu d'effets sur la gestion commune de la ressource par les communautés de recherche. Ostrom précise en effet que, pour être un " commun ", la définition des règles qui visent à maintenir la ressource dans la durée reste la prérogative des communautés de pratique. On pourrait donc imaginer que la construction de ces mesures d'impact puisse être traitée comme une activité inhérente à la pratique scientifique, sur des projets d'étude concrets par exemple.

Enfin, considérant certains effets délétères de l'économie du partage mentionnés précédemment et de la place grandissante de l'intelligence collective dans la production de la recherche et de l'innovation, il apparaît aujourd'hui nécessaire de s'interroger sur la valeur de la participation de l'usager. De nombreux auteurs soulignent aujourd'hui l'importance de la part contributive de cette production (Beraud et Cormerais, 2011 ; Coriat, 2015), soit à partir d'un "revenu contributif », pour reprendre une notion chère $\mathrm{B}$. Stiegler, soit à travers une contrepartie non marchande mesurée en temps (banque de temps par exemple) ou au moyen d'accords de réciprocité 
faisant mention d'un échange contre service. Des systèmes hybrides marchands et non marchands commencent également à émerger timidement. Au sein de Coexiscience, nous préconisons par exemple de concilier deux formes de rétribution, l'une serait issue classiquement de la vente de prestations de services (formation, conseil, contrats d'étude et de recherche, etc.), l'autre serait indexée sur le temps consacré à la gestion de la communauté. Ces régulations socioéconomiques renvoient là aussi à l'organisation des relations sociales entre les membres de la communauté, d'abord sur la base des usages qu'ils peuvent développer de la ressource et ensuite en fonction des gains matériels et symboliques qu'ils tirent de son exploitation et de sa gestion. $\grave{A}$ cet égard, les régimes d'accès à la ressource sont bien institués par les pratiques sociales et justifient le rôle prédominant de la gouvernance collective, comme une troisième et dernière condition de possibilité d'un commun scientifique.

\subsection{Instaurer une « gouvernance partagée » de la ressource}

De nombreux travaux, issus de l'économie politique, des sciences de gestion, du management des systèmes d'information ou relevant encore du concept de communautés épistémiques, se sont déjà intéressés aux différentes formes d'action collective permettant à des organisations de gérer des ressources informationnelles. À partir de l'étude d'institutions qui fonctionnent, Ostrom (1990) a mis en évidence huit principes de conception d'une gestion durable d'un commun ${ }^{10}$. Ceux-ci concernent deux exigences indissociables d'une structure coopérative ou autogérée: celle de la démocratie interne à cette organisation et celle de ses relations au reste de

\footnotetext{
10 1) Existence de limites clairement définies concernant l'objet de la communauté et de ses membres. 2) Adaptation des règles d'exploitation aux conditions locales (nature de la ressource, main-d'œuvre, matériel, etc. 3) Dispositifs de choix collectifs incluant les individus concernés par la gestion de la ressource. 4) Modalités de surveillance du comportement des individus ayant accès à la ressource. 5) Sanctions graduelles en direction des individus qui transgressent les règles. 6) Mécanismes de résolution des conflits rapides à la communauté. 7) Reconnaissance minimale par les autorités externes du droit à l'auto-organisation. 8) Imbrication des institutions locales au sein d'institutions de plus grande échelle en cas de gestion de ressources communes.
} 
Conditions d'émergence et enjeux des communs scientifiques

à partir d'une expérimentation lyonnaise

la société. Dardot et Laval (2014) envisagent davantage, à cet endroit, des formes "d'auto-gouvernement " plutôt que d'autogestion, cette terminologie laissant supposer que la direction effective de l'organisation appartient à d'autres personnes que ceux qui la "gèrent». Dans le monde académique de la recherche, la dimension d'autogouvernement des projets de libre accès à la connaissance reste relativement absente, si l'on exclut peut-être les modalités de révision des publications par les pairs. Les mouvements autour de l'open science et des sciences citoyennes, et plus récemment l'arrivée de chercheurs dans des "tiers lieux » ou dans des coopératives émanant du secteur de l'économie sociale et solidaire, semblent dessiner de nouvelles perspectives, quand bien même leurs existences restent fragiles et sont encore largement interdépendantes de l'économie classique marchande (cf. Allaire, 2013).

Les conférences de consensus, de conventions de citoyens et d'autres démarches de type "sociocratique », privilégiant davantage le consentement, représentent autant d'outils de gouvernance collective déjà éprouvés pour faciliter l'émergence d'un accord ou d'une décision collective. À cela, il convient d'ajouter d'autres formes d'encadrement qui touchent à la nécessité de préserver les valeurs partagées de la communauté (établissement de chartes, etc.), d'équilibrer les aménités au sein du groupe (mécanismes de résolution des conflits ou de contrôle, arbitrage, etc.) et de fixer une ossature managériale, réglementaire ou juridique au collectif (organisation dite "agile " ou "halocratique ", association, coopérative d'activité, etc.).

Imaginer une organisation économique alternative de production et de diffusion de la recherche gérée comme un commun, d'une part, et se situant à l'interface du secteur public et du secteur privé, d'autre part, n'est pas chose aisée dans certains pays, notamment en France, car on risque assez vite d'achopper sur la question du statut général de la fonction publique (celui des collectivités comme celui de ses agents), qui fixe les dispositions législatives et réglementaires de la recherche (droits et obligations liés au travail, à la propriété intellectuelle, etc.), voire sur de fortes résistances culturelles de confrères au sein même de l'institution. C'est pourquoi un tel dispositif ne peut se soustraire à la seconde exigence 
mentionnée plus haut, celle des relations au reste de la société. Ostrom, sans insister expressément sur le local, semble cependant faire de cette échelle de proximité un niveau structurant la gouvernance des communs. Trois de ses huit principes portent sur une nécessaire synergie avec le reste de la société. Parmi ceux-ci, on retiendra la reconnaissance des autorités externes à l'auto-organisation et l'imbrication des différentes structures partenaires dans le dispositif de gouvernance. Les villes sont aujourd'hui devenues des lieux de savoirs et d'innovation ouverte. Des fabriques urbaines aux laboratoires citoyens, ces lieux défendent le caractère endogène, incertain et bottom-up de l'innovation, souvent en réaction aux illusions technicistes des villes intelligentes ou totémiques des villes créatives (Magnaghi, 2014). Certains d'entre eux se sont d'ores et déjà imposés dans le paysage urbain grâce à des partenariats féconds avec les autorités (cf. Besson, 2016).

\section{Conclusion}

Au terme de cette contribution, il apparaît que le « commun scientifique » que nous avons tenté de circonscrire n'est pas un bien ex nihilo, mais un ensemble de procédés permettant de gérer collectivement une ressource informationnelle. Nous avons d'abord considéré le travail cognitif autour de cette information comme un processus d'apprentissage. Nous avons ensuite identifié un certain nombre de leviers économiques afin de réguler ses droits d'accès. Puis, nous avons insisté sur le volet sociopolitique de sa gouvernance. Ces procédés relèvent indistinctement du droit, des technologies, de l'économie politique, etc. II ressort de ce panorama éclectique une certaine instabilité et des négociations permanentes des mouvements en cours issus principalement des sciences participatives et de l'open science. Au-delà de ces jalons prospectifs appliqués à une expérimentation naissante, les analyses à venir sur les communs scientifiques pourraient tirer meilleur avantage de recherches situées sur la fabrique du "commun » en train de se faire, notamment sous l'angle d'une épistémologique des connaissances renouvelée. 
Conditions d'émergence et enjeux des communs scientifiques

à partir d'une expérimentation lyonnaise

\section{Références}

Allaire, G. (2013). Les communs, comme infrastructure institutionnelle de l'économie marchande. Revue de la régulation : capitalisme, institutions, pouvoirs, 14(2). Repéré à https://regulation.revues.org/10546

Beraud, P. et Cormerais, F. (2011). Économie de la contribution et innovation sociétale. Innovations, 1(34), 163-183.

Bernard, C. (2015, 6 avril). Le nombre de brevets n'arrête pas d'augmenter, mais ça ne veut pas dire que l'innovation se porte bien. SlateFR. Repéré à http://www.slate.fr/story/98417/brevets

Besson, R. (2016, 11 janvier). Les laboratoires citoyens madrilènes: la fabrique des "communs urbains". UrbaNews.fr. Repéré à https://www.urbanews.fr/2016/01/11/50396laboratoires-citoyens-madrilenes-fabrique-communsurbains/

Bollier, D. (2014). La renaissance des communs. Paris, France : Charles Léopold Mayer.

Boyle, J. (2003). The Second Enclosure Movement and the Construction of the Public Domain. Law and Contemporary Problems, 66, 33-74.

Brossaud, C. (2013). Valoriser l'innovation sociale et responsable avec les SHS: nouveau défi pour le XXI ème siècle ?. Repéré à https://halshs.archives-ouvertes.fr/halshs00832211

Brossaud, C. et Reber, B. (2010). Digital Cognitive Technologies. Epistemology and Knowledge Economy. Londres, Angleterre : ISTE-Wiley and sons.

Brossaud, C. et Fiori, S. (2018). Dossier sur les communs urbains. Repéré à http://www.metropolitiques.eu/, à paraître au second trimestre.

Callon M., Lascoumes, P. et Barthe, Y. (2001). Agir dans un monde incertain. Essai sur la démocratie technique. Paris, France : Seuil. 
Cardon, D. et Caselli, A. (2017). Qu'est-ce que le Digital Labor?. Paris, France : INA.

Castells, M. (1998). La société en réseau, vol. I. L'ère de l'information. Paris, France : Fayard.

Chesbrough, H. (2015). From Open Science to Open Innovation. Repéré à

https://www.fosteropenscience.eu/sites/default/files/pdf/ 1798.pdf

Cook-Deegan R. et Dedeurwaerdere, T. (2006). Biens communs scientifiques et recherche en sciences de la vie: structure, fonction et valeur de l'accès à la diversité génétique. Revue internationale des sciences sociales, 188(2), 317-338.

Coriat, B. (2015). Le retour des communs, la crise de l'idéologie propriétaire. Paris, France : Les liens qui libèrent.

Coutellec, L. (2012). Slow Science : pour un autre rapport aux savoirs. Repéré à

https://leocoutellec.files.wordpress.com/2012/05/slowscience-pour-un-autre-rapport-aux-savoirs-mai2012.pdf

Coutellec, L. (2015). La science au pluriel. Essai d'épistémologie pour des sciences impliquées. Paris, France : Quae.

Couture, S. (2014, 3 novembre). Science et bien commun : les transformations des pratiques scientifiques à l'ère du numérique. ritimo. Repéré à http://www.ritimo.org/Science-etbien-commun-les-transformations-des-pratiquesscientifiques-a-I-ere

Dardot, P. et Laval, C. (2014). Commun. Essai sur la révolution au $X X I^{\ominus}$ siècle. Paris, France : La Découverte.

De Moor, T. (2013). Co-operating for the future: Inspiration from the European past to develop public-collective partnerships and intergenerational co-operatives. Dans S. Baily, G. Farrell et U. Mattei (dir.), Protecting future generations through commons (Trends in social cohesion, series 26) (p. 81-104). Strasbourg, France : Council of Europe Publishing. 
Conditions d'émergence et enjeux des communs scientifiques

à partir d'une expérimentation lyonnaise

Fayet, S. (2013, 15 novembre). «Données » de la recherche, les mal-nommées. Urfist Info. Repéré à

http://urfistinfo.hypotheses.org/2581

Feenberg, A. (2010). Between Reason and Experience: Essays in Technology and Modernity. Cambridge, MA : MIT Press.

Hess, C. et Ostrom E. (2003). Ideas, Artifacts and Facilities: Information as a Common-Pool Resource. Law and contemporary problems, 66, 111-145.

Hess, C. et Ostrom E. (2006). Understanding knowledge as a Commons: From theory to practice. Cambridge, MA : MIT Press.

Latour, B. (1988). Science in action, How to Follow Scientists and Engineers through Society. Cambridge, MA: Harvard University Press.

Lebouc, M.-F. et Chartier A. (2015). Sciences de gestion : comment la quête d'excellence freine la libre circulation des savoirs. Éthique publique, 17(2). Repéré à https://ethiquepublique.revues.org/2248

Le Crosnier, H. (2015). Une introduction aux communs de la connaissance. Caen, France : C\&F Éditions.

Leclerc, O. (2017). Savants, artistes, citoyens : tous créateurs ? Québec, Québec : Éditions science et bien commun.

Leroi-Gourhan, A. (1964). Le Geste et la Parole. Technique et langage, vol. 1. Paris, France : Albin Michel.

Magnaghi, A. (2014). La biorégion urbaine. Petit traité sur le territoire " bien commun ». Paris, France : Eterotopia.

Mille, A. (2013). De la trace à la connaissance à l'ère du Web. Intellectica, 1(59), 89-113.

Mille, A. et Magué, J.-P. (2012). Le Web: la révélation documentaire ? Manifeste pour un fait documentaire sur le web. Dans B. Stiegler (dir.), Confiance, croyance, crédit dans les mondes industriels. Limoges, France: Fyp. Repéré à https://hal.archives-ouvertes.fr/hal-00716759

Nicolas-Le Srat, P. (2016). Le travail du commun. Paris, France : Éditions du commun. 
Moulier-Boutang, Y. (2007). Le capitalisme cognitif. La Nouvelle Grande Transformation. Paris, France : Amsterdam.

Noyer, J.-M. et Carmes, M. (2014). L'irrésistible montée de l'algorithmique : méthodes et concepts en SHS. Les Cahiers du numérique, 10(4), 63-110.

Ostrom, E. (1990). Governing the Commons. Cambridge, MA : Cambridge University Press.

Pellé, S. et B. Reber (2016). Éthique de la recherche et innovation responsable. Londres, Angleterre : ISTE Éditions.

Peugeot, V. (2016, 29 septembre). Facilitatrice, protectrice, instituante, contributrice : la loi et les communs. Citoyenneté dans la société numérique. Repéré à https://vecam.org/Facilitatrice-protectrice-instituantecontributrice-la-loi-et-les

Penaranda, A., Amabile, S. et Haller, C. (2017). Management des biens communs de la connaissance: principes de conception et gouvernance de l'action collective. Systèmes d'information et management, 28. Repéré à

https://www.researchgate.net/publication/320766477_Manag ement_des_biens_communs de_la_connaissance_principes de_conception_et_gouvernance_de_l'action_collective

Rumpala, Y. (2014, 17 décembre). Une nouvelle forme d'atelier en versions " high-tech »?. Le blog de Yannick Rumpala. Repéré https://yannickrumpala.wordpress.com/2014/12/17/unenouvelle-forme-datelier-en-version-high-tech/

Storup, B., Millot, G. et Neubauer C. (2013). La recherche participative comme mode de production des savoirs. Un état de lieux des pratiques en France. Paris, France: Fondation Sciences citoyennes.

Testart, J. (2015). L'humanitude au pouvoir. Comment les citoyens peuvent décider du bien commun. Paris, France: Seuil.

Vecam (2011). Libres savoirs, les biens communs de la connaissance. Produire collectivement, partager et diffuser la connaissance au $X X I^{e}$ siècle. Caen, France : C\&F Éditions. 